Article

\title{
Hypoxia Inhibits Subretinal Inflammation Resolution Thrombospondin-1 Dependently
}

\author{
Sara Touhami ${ }^{1,2}$, Fanny Béguier ${ }^{1}$, Tianxiang Yang ${ }^{1}$, Sébastien Augustin ${ }^{1}$, Christophe Roubeix ${ }^{1}$, \\ Frederic Blond ${ }^{1} \mathbb{D}$, Jean Baptiste Conart 1,3, José Alain Sahel 1,4, Bahram Bodaghi ${ }^{2}$, Cécile Delarasse ${ }^{1}$, \\ Xavier Guillonneau ${ }^{1}$ (D) and Florian Sennlaub ${ }^{1, *}$
}

\section{check for}

updates

Citation: Touhami, S.; Béguier, F.;

Yang, T.; Augustin, S.; Roubeix, C.;

Blond, F.; Conart, J.B.; Sahel, J.A.;

Bodaghi, B.; Delarasse, C.; et al.

Hypoxia Inhibits Subretinal

Inflammation Resolution

Thrombospondin-1 Dependently. Int. J. Mol. Sci. 2022, 23, 681. https:// doi.org/10.3390/ijms23020681

Academic Editors: Hiroshi Tomita and Janusz Blasiak

Received: 30 September 2021

Accepted: 4 January 2022

Published: 8 January 2022

Publisher's Note: MDPI stays neutra with regard to jurisdictional claims in published maps and institutional affiliations.

Copyright: (C) 2022 by the authors. Licensee MDPI, Basel, Switzerland. This article is an open access article distributed under the terms and conditions of the Creative Commons Attribution (CC BY) license (https:// creativecommons.org/licenses/by/ $4.0 /)$
1 Institut de la Vision, Sorbonne Université, INSERM, CNRS, 75012 Paris, France; saratouhami@gmail.com (S.T.); fanny.beguier@gmail.com (F.B.); tianxiang.yang@inserm.fr (T.Y.); sebastien.augustin@inserm.fr (S.A.); chris.roubeix@gmail.com (C.R.); frederic.blond@inserm.fr (F.B.); jbconart@hotmail.com (J.B.C.); j.sahel@gmail.com (J.A.S.); cecile.delarasse@sorbonne-universite.fr (C.D.); xavier.guillonneau@inserm.fr (X.G.)

2 Ophthalmology Department, Pitié Salpêtrière University Hospital, Sorbonne Université, AP-HP, 75013 Paris, France; bahram.bodaghi@aphp.fr

3 Department of Ophthalmology, University Hospital, 54000 Nancy, France

4 CHNO des Quinze-Vingts, INSERM-DGOS CIC 1423, 75012 Paris, France

* Correspondence: florian.sennlaub@inserm.fr

Abstract: Hypoxia is potentially one of the essential triggers in the pathogenesis of wet age-related macular degeneration (wetAMD), characterized by choroidal neovascularization (CNV) which is driven by the accumulation of subretinal mononuclear phagocytes (MP) that include monocytederived cells. Here we show that systemic hypoxia $\left(10 \% \mathrm{O}_{2}\right)$ increased subretinal MP infiltration and inhibited inflammation resolution after laser-induced subretinal injury in vivo. Accordingly, hypoxic $\left(2 \% \mathrm{O}_{2}\right)$ human monocytes $(\mathrm{Mo})$ resisted elimination by RPE cells in co-culture. In Mos from hypoxic mice, Thrombospondin 1 mRNA (Thbs1) was most downregulated compared to normoxic animals and hypoxia repressed Thbs- 1 expression in human monocytes in vitro. Hypoxic ambient air inhibited MP clearance during the resolution phase of laser-injury in wildtype animals, but had no effect on the exaggerated subretinal MP infiltration observed in normoxic Thbs1 $1^{-/-}$-mice. Recombinant Thrombospondin 1 protein (TSP-1) completely reversed the pathogenic effect of hypoxia in Thbs1 $1^{-/-}$-mice, and accelerated inflammation resolution and inhibited CNV in wildtype mice. Together, our results demonstrate that systemic hypoxia disturbs TSP-1-dependent subretinal immune suppression and promotes pathogenic subretinal inflammation and can be therapeutically countered by local recombinant TSP-1.

Keywords: age-related macular degeneration; hypoxia; macrophages; mononuclear phagocytes; choroidal neovascularization; thrombospondin 1

\section{Introduction}

Age-related macular degeneration (AMD) is characterized by sizeable deposits of lipoproteinaceous debris called soft drusen (early AMD), choroidal neovascularisation (wet $\mathrm{AMD}$, late form), or by an extending lesion of the retinal pigment epithelium (RPE) and photoreceptors (geographic atrophy, GA, late form) [1].

A common feature of early and both advanced forms of AMD is their association with chronic accumulation of mononuclear phagocytes (MPs) in the subretinal space, which is physiologically immunosuppressive and devoid of MPs, at least in part due to the presence of immune-suppressive retinal pigment epithelium cells (RPE) [2]. The MP infiltrate is composed of displaced resident macrophages $(\mathrm{M} \varphi)$, such as microglial cells (MCs) and choroidal $\mathrm{M} \varphi \mathrm{s}$, and monocyte (Mo)-derived inflammatory $\mathrm{M} \varphi \mathrm{s}$ (iM $\varphi s$ ) [3]. Functional studies in animal models show that the subretinal accumulation of iM $\varphi$ s play a critical 
role in neovascularization and photoreceptor degeneration that characterize late AMD [2]. However, the reasons for the establishment of non-resolving pathogenic inflammation in AMD is not clear, but may be found in the downstream consequences of AMD-risk factors.

AMD is a common, complex disease that results from the interplay of age, environmental risk factors and genetic variants [2]. We recently demonstrated that the two main genetic AMD-risk variants, CFH Y402H and a haplotype of 10q26, curb TSP-1 activation of CD47 that is necessary for homeostatic MP elimination and the resolution of acute inflammation in the subretinal space [4,5]. Accordingly, TSP-1 deletion leads to increased subretinal inflammation accompanied by increased CNV in laser-injured mice [5-7].

Hypoxia, among other contributing mechanisms such as oxidative stress and dysfunctional autophagy, has long been supposed to be an important trigger of AMD [8]. Late AMD, in particular wet AMD is preceded by choriocapillary dropout, reduced ocular blood flow, and drusen deposits that impede with the oxygen supply from the choroid to RPE and photoreceptors [9]. From a systemic point of view, AMD is associated with hypertension, atherosclerosis, cardiovascular disease [10], and emphysema [11] that are all associated with systemic hypoxia [12]. Indeed, hypoxia can activate MPs and induce inflammatory cytokines such as CCL2, TNF $\alpha$, IL-1 $\beta$, and IL-6 [13], all shown to be implicated in subretinal inflammation [3,14-18]. Pre-activation of circulating Mos promoting subretinal chronic inflammation could therefore be one of the mechanisms that link systemic hypoxia to AMD.

Here we show that TSP-1 is the most downregulated transcript in monocytes of mice exposed to systemic hypoxia, which strongly reduces the elimination of subretinal MPs. Using TSP-1-deficient mice and recombinant TSP-1, we demonstrate that the hypoxia induced inhibition of MP elimination is dependent on downregulation of TSP-1. Importantly, we demonstrate that intravitreal injections of recombinant TSP-1 completely reversed the pathogenic effect of hypoxemia, accelerated inflammation resolution, and inhibited CNV in vivo.

\section{Material and Methods}

\subsection{Laser-Injury Model, Intravitreal Injections, and Hypoxia}

Male C57BL6/J animals (Janvier-labs, Le Genest-Saint-Isle, France), aged 10 to 12 weeks, were used in this study. Wild type and Thbs1-/ oratories. All mice were either negative or backcrossed to eliminate the Pde6b ${ }^{\text {rd1 }}$, Gnat2 ${ }^{\text {cpfl3 }}$, and $\mathrm{Crb} 1^{r d 8}$ mutations. Animals were housed in the animal facility under specific pathogenfree condition, in a 12/12 h light/dark (100-500 lux) cycle with water and normal diet food available ad libitum. Laser-coagulations were performed with a Vitra Laser (Quantel Medical, Coumon -d'Auvergne, France) mounted on a surgical microscope as previously described [19]. Briefly, four equidistant impacts (532 nm, $450 \mathrm{~mW}, 50 \mathrm{~ms}$, and $250 \mu \mathrm{m}$ ) per eye were applied in the mid-periphery. In certain experiments the eyes were intravitreally injected 4 and 7 days after photocoagulation using glass capillaries (Eppendorf, Hamburg, Germany) and a microinjector, with either $2 \mu \mathrm{L}$ of PBS, or recombinant human TSP-1 (R\&D Systems, Minneapolis, MN, USA, $10 \mu \mathrm{g} / \mathrm{mL}=80 \mathrm{nM}$ ). The mice were sacrificed at the indicated time points and immune-stained RPE/Choroidal flatmounts were analyzed. Ambient hypoxia $10 \% \mathrm{O}_{2} / 90 \% \mathrm{~N}_{2}$ was administered using an Oxycycler for the indicated times. Control mice breathing normoxic air were housed in the same room under identical conditions. For qPCR analyses, mice were exposed to hypoxia for $40 \mathrm{~h}$ then euthanized and cell sorting was performed immediately after the end of the hypoxic challenge as previously described [4]. All experimental procedures were approved by the Ministere de l'éducation nationale, de l'enseignement supérieur et de la recherche (APAFIS\#2636-2015110914346299v2).

\subsection{Monocyte RPE Co-Cultures}

Monocyte/RPE co-cultures were performed as previously described [16,17]. Briefly, primary porcine RPE cells were seeded at a density of 75000 cells/well in DMEMFCS20\%-PS1\% and cultured for 4 days before use. We previously characterized these 
primary RPE cell cultures, showing that they form tight junctions and phagocytose photoreceptor outer segments, stain positive for $\mathrm{ZO} 1$, and express retinol dehydrogenase 5 , transthyretin, transferrin $[16,17]$. Monocytes were prepared from human blood from healthy volunteers after written informed consent (approved by the Direction Générale pour la Recherche et l'Innovation of the Ministère de l'Enseignement et de la Recherche (Dossier $\mathrm{n}^{\circ} 14.007$ ) and by the Commission Nationale de $\mathrm{l}^{\prime}$ Informatique et des Libertés (N/Ref.: IFP/MKE/AR144088)). Briefly, CD14 ${ }^{+}$peripheral blood Mos were isolated by negative selection using the EasySep Human Monocyte Enrichment Cocktail (StemCell Technologies, Saint Egrève, France) as previously described [5]. In vitro hypoxia conditions were created using a hypoxia incubator chamber (Stemcell technologies) filled with $2 \%$ $\mathrm{O}_{2}, 5 \% \mathrm{CO}_{2}$, and $93 \% \mathrm{~N}_{2}$, and de-pressurized after $1 \mathrm{~h}$ of culture (hypoxic conditions) and compared to standard normoxic cell culture conditions $\left(5 \% \mathrm{CO}_{2}, 19.9 \% \mathrm{O}_{2}, 75.1 \% \mathrm{~N}_{2}\right)$. RPE cells were serum-starved for $24 \mathrm{~h}$ prior to co-culture with hMos (100 000 cells/well) under normoxic or hypoxic conditions for $24 \mathrm{~h}$. In some experiments each cell type was pre-incubated for $24 \mathrm{~h}$ in normoxic or hypoxic conditions for $24 \mathrm{~h}$ followed by $24 \mathrm{~h}$ normoxic co-culture. Ultra-low adherence surface 96-well culture plates (Corning, Amsterdam, Netherlands) were used throughout to permit Mo transfer after $24 \mathrm{~h}$ of pre-incubation by gently pipetting them off the low adherence culture plates. After culture, the plates were fixed in $4 \%$ PFA and stained with goat anti-human OTX2 (R\&D, 1/500) (specific of RPE cells) and rabbit anti-human hematopoietic transcription factor PU-1, 1/200 (specific of mononuclear phagocytes) and nuclei were counterstained with Hoechst (1/1000, SigmaAldrich, Saint-Quentin-Fallavier, France) as previously described [16,17]. Twenty-five fields per well were analyzed and recorded using the Arrayscan software (HCS iDev Cell Analysis Software, Thermo Fisher Scientific, Les Ulis, France).

\subsection{Gene Expression Analysis}

For whole transcriptome analysis, Ly6 $\mathrm{C}^{\text {high }}$ bone-marrow monocytes sorted from normoxic and $40 \mathrm{~h}$ hypoxic mice were prepared. After cell lysis, RNA was extracted using the Qiagen RNA Mini Kit with RNase (ribonuclease)-free DNase (deoxyribonuclease) I digestion. RNA quality and quantity were evaluated using BioAnalyzer 2100 with the RNA 6000 pico Kit (Agilent Technologies, Leuven, Belgique). RNA sequencing libraries were constructed from $200 \mathrm{ng}$ of total RNA using a modified TruSeq RNA Sample preparation kit protocol. Pass-filtered reads (using Trimmomatic) were mapped using HiSAT2 and aligned to human reference genome GRCh38.95 [20]. The count table of the gene features was obtained using HTSeq. Normalization and differential expression analysis values were computed with DESeq2 [21]. TPM were determined using Libinorm using htseq mode [22]. Protein coding mRNAs with greater than 100 TPM in the normoxic group and a false discovery rate $<0.05$ were selected. For reverse transcription and real-time quantitative polymerase chain reaction (RT-qPCR) total RNA from human and mouse Mos, mouse MCs, and eye-cup were extracted and PCR was performed using StepOne Plus real-time PCR system (Applied Biosystems) as previously described [4]. Results were normalized using house-keeping gene RPS26. PCRs were performed in 45 cycles of $15 \mathrm{~s}$ at $95^{\circ} \mathrm{C}, 45 \mathrm{~s}$ at $60^{\circ} \mathrm{C}$. Primers for RT-PCR were purchased from IDT technology (primer sequences at request).

\subsection{Immunohistochemistry, $\mathrm{CNV}$ and $\mathrm{MPs}$ Quantification}

RPE and retinal flatmounts were stained and quantified as previously described [3] using polyclonal rabbit anti- IBA-1 (Wako, Neuss, Germany) and goat anti-mouse ColIV (Biorad, Mitry-Mori, France; 1/100) and appropriate secondary antibodies and counterstained with Hoechst if indicated. Preparations were observed with a fluorescence microscope (DM5500, Leica, Nanterre, France).

\subsection{Statistical Analyses}

Graph Pad Prism 7 (GraphPad Software) was used for data analysis and graphic representation. All values are reported as mean \pm SEM. Statistical analyses were performed 
by Mann-Whitney test for comparison of mean values. The $n$ and $p$-values are indicated in the figure legends.

\section{Results}

\subsection{Hypoxia Increases the Infiltration of Subretinal MPs after Laser-Injury}

The subretinal space can be visualized by flat-mount preparations and is physiologically avascular and devoid of IBA $1^{+} \mathrm{MPs}$. Laser-injury induces the infiltration of subretinal IBA $1^{+} \mathrm{MPs}$, with a maximal recruitment three to four days after the injury, followed by an inflammation resolution phase characterized by dwindling MP numbers and choroidal neovascularization (CNV) formation [14]. 50-70\% of the lesional MPs are derived from circulating Mos and the remainder from resident macrophages (microglial cells and choroidal macrophages) $[5,23]$. Depletion of circulating Mo $[5,23,24]$ and inhibition of Morecruitment [25-29] very significantly inhibits CNV formation. Importantly, the infiltrating MPs are observed within the injured tissue in close contact with the forming CNV, but also in the subretinal space, adjacent to physiologically immunosuppressive RPE cells, that are potent inducers of MP death [4,14-16].

To evaluate the influence of hypoxia on subretinal neuro-inflammation we exposed laser-injured mice to $10 \%$ or $20.9 \%$ of ambient oxygen. As young, otherwise healthy, mice quickly adapt to hypoxia, increasing their hematocrit [30] we either exposed the mice during the early phase $(\mathrm{d} 0-4)$ or during the inflammation resolution phase, when MP elimination exceeds the recruitment rate (d4-10; experimental design Figure 1A). Quantification on RPE/choroidal flatmounts of subretinal IBA- $1^{+}$(green) MPs on Collagen$4^{+}\left(\mathrm{Coll}^{+}\right.$, red) CNV and on the surrounding RPE (0-500 $\mu \mathrm{m}$ from the lesion) revealed that hypoxia did not significantly alter the density of subretinal MPs in the RPE-denuded lesions, directly adjacent to the endothelial cells of the CNV. However, it significantly increased the accumulation of IBA $1^{+}$MPs that accumulate on the immunosuppressive RPE at $\mathrm{d} 4$ (190\% increase) and induced a continued accumulation of MPs from d4 to d10, when the MP accumulation started to resolve in normoxic mice (460\% increase at day 10; Figure 1B-D). Additionally, the size of Coll4 ${ }^{+} \mathrm{CNV}$ was significantly increased at d10 (Figure 1E).

To test whether hypoxia altered RPE-induced Mos elimination in vitro, we incubated equal numbers of peripheral blood Mos with primary RPE cells for $24 \mathrm{~h}$ under normoxic $\left(20.9 \% \mathrm{O}_{2}\right)$ or hypoxic $\left(2 \% \mathrm{O}_{2}\right)$ conditions and stained the co-culture with an anti-PU1- and anti OTX2-antibodies that allows the differentiation of PU1 ${ }^{+}$OTX2 ${ }^{\text {neg }}$ Mo and OTX $2^{+} \mathrm{PU} 1^{\text {neg }} \mathrm{RPE}$ cells in this cell culture system. Automated quantification of PU $1^{+} \mathrm{Mos}$ and OTX $2^{+} \mathrm{RPE}$ cells revealed that hypoxic conditions significantly increased the number of surviving Mos after $24 \mathrm{~h}$ of co-culture, but had no effect on the number of RPE cells (Figure 1E). Interestingly, $24 \mathrm{~h}$ hypoxic pre-incubation of Mo (Figure 1F), but not RPE cells (Figure 1G), followed by $24 \mathrm{~h}$ normoxic co-culture, similarly increased the number of remaining $\mathrm{PU}^{+}$Mos.

Taken together, our experiments show that systemic hypoxia, induced by reduced ambient air oxygen, is sufficient to significantly increase subretinal MP accumulation and CNV after laser-injury. Interestingly, these differences were observed in the RPE-adjacent MP population surrounding the injury only and were more pronounced when the hypoxia was administered during the inflammation resolution phase. These results suggested that hypoxia interferes with the RPE-induced MP elimination. Our in vitro results show that hypoxia did not diminish immunosuppressive signals from the RPE but significantly increased Mos resistance to RPE-induced elimination. 
A
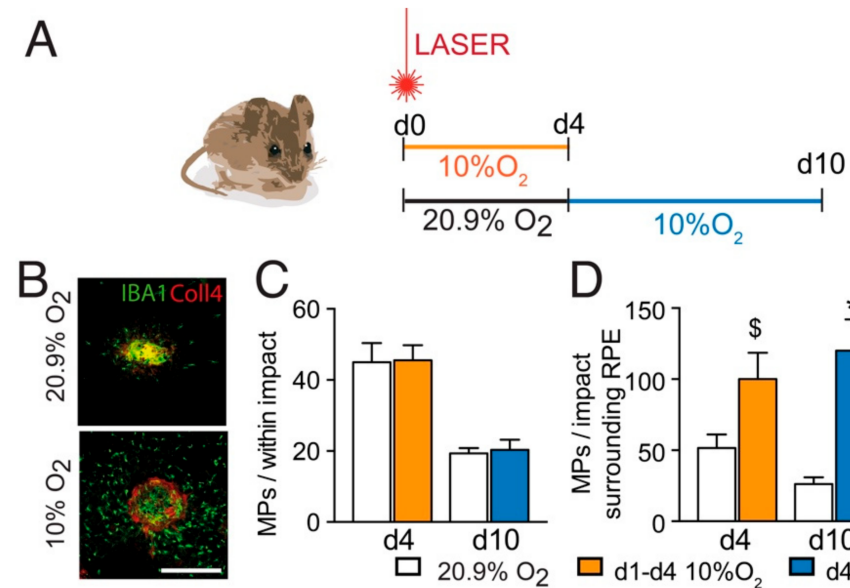

D
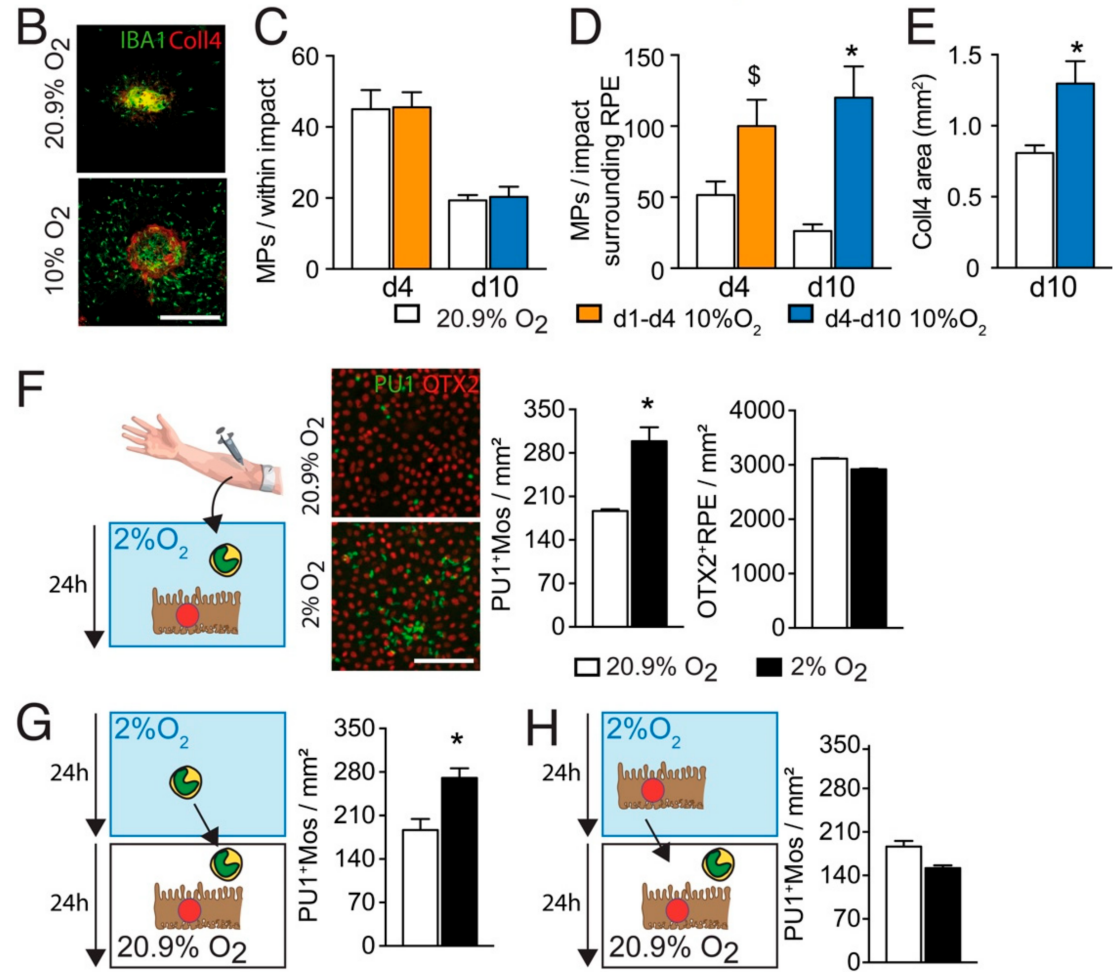

Figure 1. Hypoxia increases Mo resistance to RPE-induced elimination. (A) schematic representation of the experiments: mice were laser injured and either exposed to $10 \% \mathrm{O}_{2}$ hypoxia from $\mathrm{d} 0-\mathrm{d} 4$ and evaluated for the presence of subretinal IBA $1^{+} \mathrm{MPs}$ at $\mathrm{d} 4$ or exposed to $10 \% \mathrm{O}_{2}$ hypoxia from $\mathrm{d} 4-\mathrm{d} 10$ and evaluated for MPs and the extent of Collagen $4^{+} \mathrm{CNV}$ at d10. Mice raised in normal $20.9 \%$ ambient $\mathrm{O}_{2}$ served as controls. (B) Representative images of Collagen 4 (Coll4; red) and IBA-1 (green) immuno- stained RPE/choroid flatmounts of 10 days post laser-injured normoxic and hypoxic (d4-d10) mice. (C,D) Quantification of the density of subretinal IBA-1+ MPs per impact: (B) directly within the RPE-denuded lesion and (C) counted at a distance of $0-500 \mu \mathrm{m}$ to Coll4 ${ }^{+} \mathrm{CNV}$ on the apical side of the RPE surrounding the lesion at $\mathrm{d} 4$ and $\mathrm{d} 10$ of normoxic and hypoxia-exposed 2-month-old mice (room air white columns, $10 \% \mathrm{O}_{2}$ hypoxia $\mathrm{d} 0$ - $\mathrm{d} 4$ orange columns; $10 \% \mathrm{O}_{2}$ hypoxia $\mathrm{d} 4-\mathrm{d} 10$ blue columns) ( $n=9-10$ eyes; $\$ p=0.0503 ;{ }^{*} p<0.0001$ Mann-Whitney versus their normoxic controls). (E) Quantification of the Coll4 ${ }^{+} \mathrm{CNV}$ surface at $\mathrm{d} 10$ of normoxia and hypoxia-exposed (d4-d10) laserinjured mice ( $n=9-10$ eyes; ${ }^{*} p=0.0060$ Mann-Whitney versus normoxic control). (F) Representative pictures of PU-1 OTX-2 co-stained co-cultures of PU1 ${ }^{+}$human monocytes (green) and OTX-2 ${ }^{+} \mathrm{RPE}$ cells (red) under normoxic- $\left(20 \% \mathrm{O}_{2}\right.$; white columns) and hypoxic- $\left(2 \% \mathrm{O}_{2}\right.$; black columns) conditions and their automated quantifications after $24 \mathrm{~h}$ co-culture $\left(n=5\right.$ wells; ${ }^{*} p=0.0079$ Mann-Whitney versus the normoxic condition), (G) quantifications of PU1 ${ }^{+}$human monocytes after $24 \mathrm{~h}$ of hypoxic (black column) or normoxic (white column) pre-incubation of Mo followed by $24 \mathrm{~h}$ of Mo/RPE coculture ( $n=5$ wells; ${ }^{*} p=0.0079$ Mann-Whitney versus the normoxic condition) $(\mathbf{H})$ automated quantifications of $\mathrm{PU} 1^{+}$human monocytes after $24 \mathrm{~h}$ of hypoxic (black column) or normoxic (white column) pre-incubation of RPE followed by $24 \mathrm{~h}$ of Mo/RPE coculture. The in vivo results presented in (B-E) summarize two independently carried out experiments, the in vitro experiments $(\mathbf{F}-\mathbf{H})$ were repeated a minimum of five times and gave similar results. All values are reported as mean \pm SEM. IBA-1: ionized calcium adapter molecule 1; Coll4: Collagen 4; PU1: hematopoietic transcription factor; OTX-2: Orthodenticle Homeobox 2; scale bar A $=400 \mu \mathrm{m}, \mathrm{E}=500 \mu \mathrm{m}$. 


\subsection{Hypoxia Decreases Thbs1-Expression in Mos}

To identify potential hypoxia-induced downstream mediators of increased Mos resistance to RPE-induced elimination we sequenced the transcriptome of FACS sorted

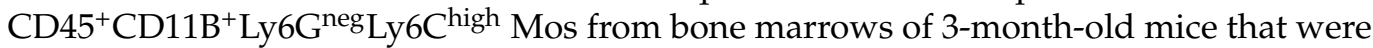
raised in room-air or had been exposed to $10 \%$ ambient $\mathrm{O}_{2}$ for $40 \mathrm{~h}$ (Figure $2 \mathrm{~A}$ ). A $40 \mathrm{~h}$ exposure time was chosen for this experiment as normal mice quickly adapt to hypoxic conditions and to detect differences that occur prior to the inflammatory changes observed in Figure 1. In the transcripts that are robustly expressed in normoxic Mo (>100 transcripts per million), our analysis identified 18 transcripts that were more than two-fold overexpressed and 19 transcripts that were more than two-fold underexpressed in Mos from hypoxic mice compared to Mos from room air raised mice.

\section{A most down and up-regulated genes in Ly6 $\mathrm{C}^{\text {high }} \mathrm{BMMs} 10 \% \mathrm{O} 2$ versus $20,9 \% \mathrm{O} 2$ exposed mice}
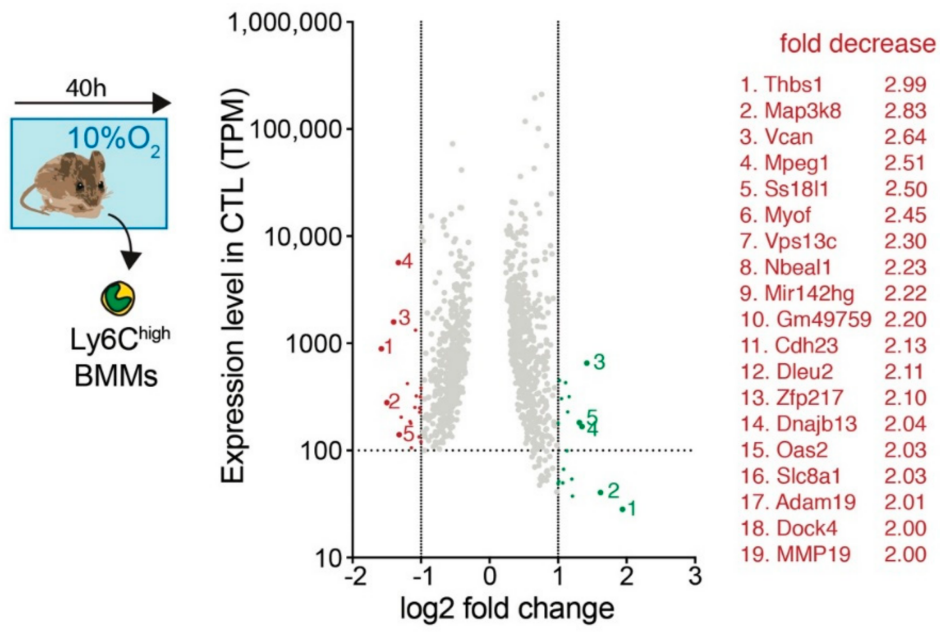

\begin{tabular}{lr}
\multicolumn{2}{|c}{ fold increase } \\
1. Stfa1 & 3.84 \\
2. Fbp1 & 3.07 \\
3. Mmp9 & 2.68 \\
4. Cirbp & 2.55 \\
5. Kit & 2.48 \\
6. Prnp & 2.31 \\
7. Hsd17b7 & 2.30 \\
8. Vcam1 & 2.23 \\
9. Tfrc & 2.20 \\
10. Tinagl1 & 2.19 \\
11. Mogat2 & 2.16 \\
12. Abcg2 & 2.11 \\
13. Tmem119 & 2.10 \\
14. Il18rap & 2.07 \\
15. Mapk13 & 2.03 \\
16. Dapk2 & 2.01 \\
17. Pheta2 & 2.00 \\
18. Cxcr2 & 2.00
\end{tabular}

B mouse samples in vivo
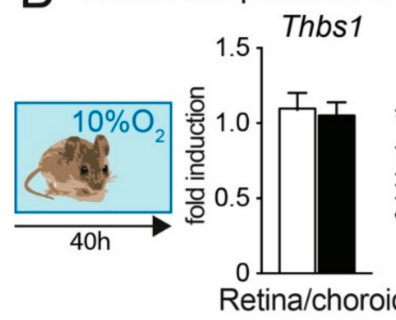

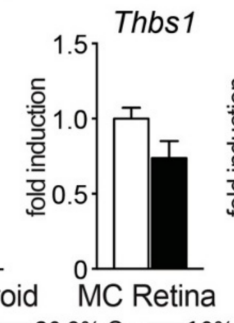

$\square 20.9 \% \mathrm{O}_{2}$

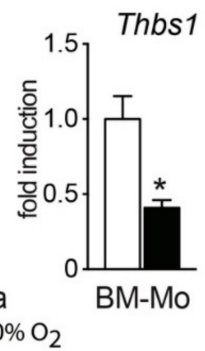

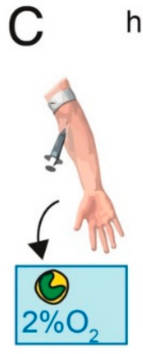

$\mathrm{hMo}$ in vitro

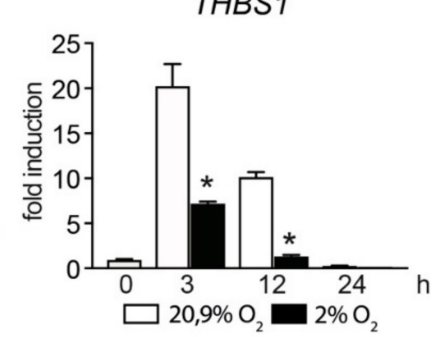

Figure 2. Hypoxia decreases Thbs1- expression in Mos. (A) Scatter dot blot of the protein coding mRNAs of FACS sorted CD45 ${ }^{+} \mathrm{CD}_{11 B^{+}} \mathrm{Ly} 6 \mathrm{G}^{\text {neg }} \mathrm{Ly} 6 \mathrm{C}^{\text {high }}$ Mos from bone marrows of five 3-month-old mice that were raised in room-air or five age-matched mice that had been exposed to $10 \%$ ambient $\mathrm{O}_{2}$ for $40 \mathrm{~h}$. Only transcripts with a TPM greater than 100 in the normoxia group and a false discovery rate (FDR) smaller than 0.05 are depicted. The transcripts are plotted according to their expression levels ( $y$-axis) and the $\log 2$-fold induction by hypoxia ( $x$ axis). The identified 18 transcripts that were more than two-fold overexpressed and the 19 transcripts that were more than two-fold underexpressed in Mos from hypoxic mice compared to Mos from room air raised mice are indicated. (B) Quantitative RT-PCR of Retina/RPE/Choroid, magnet sorted retinal microglial cells (MC), and magnet sorted bone marrow monocytes (BM-Mo) from room-air-(white columns) and 40 h hypoxia( $10 \% \mathrm{O}_{2}$; black columns) exposed mice. ( $n=5$ mice/group ${ }^{*} p=0.0032$; Mann-Whitney versus the normoxic control). (C) Quantitative RT-PCR of human Mo exposed to normoxic culture conditions (white columns) or the indicated hours of $2 \% \mathrm{O}_{2}$ (black columns) ( $n=5$ wells/group, representative of two independent experiments, ${ }^{*} p=0.0079$ (3 h) and 0.0159 (12 h); Mann-Whitney versus the normoxic control). All values are reported as mean \pm SEM. Thbs1: Thrombospondin 1. 
Interestingly, the most downregulated gene in Ly $6^{\text {high }}$ Mos from hypoxic animals was thrombospondin 1 (Thbs-1, TSP-1) that we previously showed is necessary for homeostatic MP elimination and the resolution of acute inflammation in the subretinal space $[4,5]$. Quantification by RT-PCR of Thbs1-mRNA on extracts from retina/RPE/choroid, magnetsorted retinal MCs, and magnet-sorted bone-marrow Mos from room-air control animals and mice exposed for $40 \mathrm{~h}$ to $10 \% \mathrm{O}_{2}$ hypoxia revealed that the hypoxic conditions did not alter the expression in the chorioretinal tissue or resident MCs, but confirmed the significant downregulation of Thbs1- transcription in Ly ${ }^{\text {high }}$ Mos (Figure 2B). Additionally, the strong induction of THBS1 in freshly ( $3 \mathrm{~h}$ and $12 \mathrm{~h}$ ) cultured human Mos (hMos) was significantly blunted when the early differentiating hMos were cultured in $2 \% \mathrm{O}_{2}$ (Figure $2 \mathrm{C}$ ).

These results suggest that Mo exposed to hypoxia in vitro and from hypoxic animals (Figure 1) might have a reduced capacity to activate their CD47 receptor, due to decreased TSP-1-expression, necessary for their RPE-induced elimination from the subretinal space.

\subsection{TSP-1 Is Necessary for Hypoxia-Induced Inhibition of Inflammation Resolution after Laser-Injury}

To evaluate whether TSP-1 is required for hypoxia to impair inflammation resolution we submitted wildtype-, and Thbs $1^{-/-}$- mice to laser injury and exposed the mice to ambient hypoxia during inflammation resolution (d4-d10) or kept them in room air. In room air raised mice, the quantification of IBA- $1^{+}$(green) MPs (counted on the RPE at a distance of $0-500 \mu \mathrm{m}$ to Coll4 ${ }^{+} \mathrm{CNV}$, red staining) on RPE/choroidal flatmounts at d10, revealed a significantly greater number of subretinal MPs in Thbs $1^{-/-}$-mice (Figure 3A) in accordance with their role in subretinal MP elimination we previously described in laser-injury [5], and in subretinal adoptive transfer experiments, after a light challenge and with age [4]. As shown in Figure 1, hypoxia during inflammation resolution significantly increased the number of subretinal MPs in wildtype-mice at d10, to numbers observed in Thbs $1^{-1-}$-mice kept in room-air. However, hypoxia failed to further increase the elevated levels in Thbs1 $1^{-/-}$-mice, showing the necessity of the presence of TSP- 1 for systemic hypoxia to impede inflammation resolution in our experimental conditions. In terms of Coll4 ${ }^{+} \mathrm{CNV}$ formation in room-air exposed animals, we observed increased CNV formation in Thbs $1^{-/-}$-mice compared to wildtype mice as previously described $[5,7]$, but again failed to alter the extent of $\mathrm{CNV}$ in Thbs1 $1^{-/-}$-mice (Figure 3B).

Next, we next injected wildtype mice intra-vitreally four days after laser-injury with PBS or recombinant TSP-1 (rTSP-1), and exposed them to hypoxia until sacrifice at d10. Quantification of IBA- $1^{+}$(green) MPs surrounding the lesion on RPE/choroidal flatmounts at $\mathrm{d} 10$, revealed that the local injection of rTSP-1 completely reversed the effect of hypoxia during the inflammation resolution phase (Figure 3C). Concomitantly, the intravitreal administration of rTSP-1 significantly reduced the exaggerated $\mathrm{CNV}$ in this condition (Figure 3D).

These experiments strongly suggest that the promotion of pathogenic subretinal inflammation by systemic hypoxia is at least in part mediated by the downregulation of TSP-1, which inhibits subretinal MP elimination. They also demonstrate that the pro-inflammatory effect of systemic hypoxia can be reversed pharmacologically by local drug administration. 

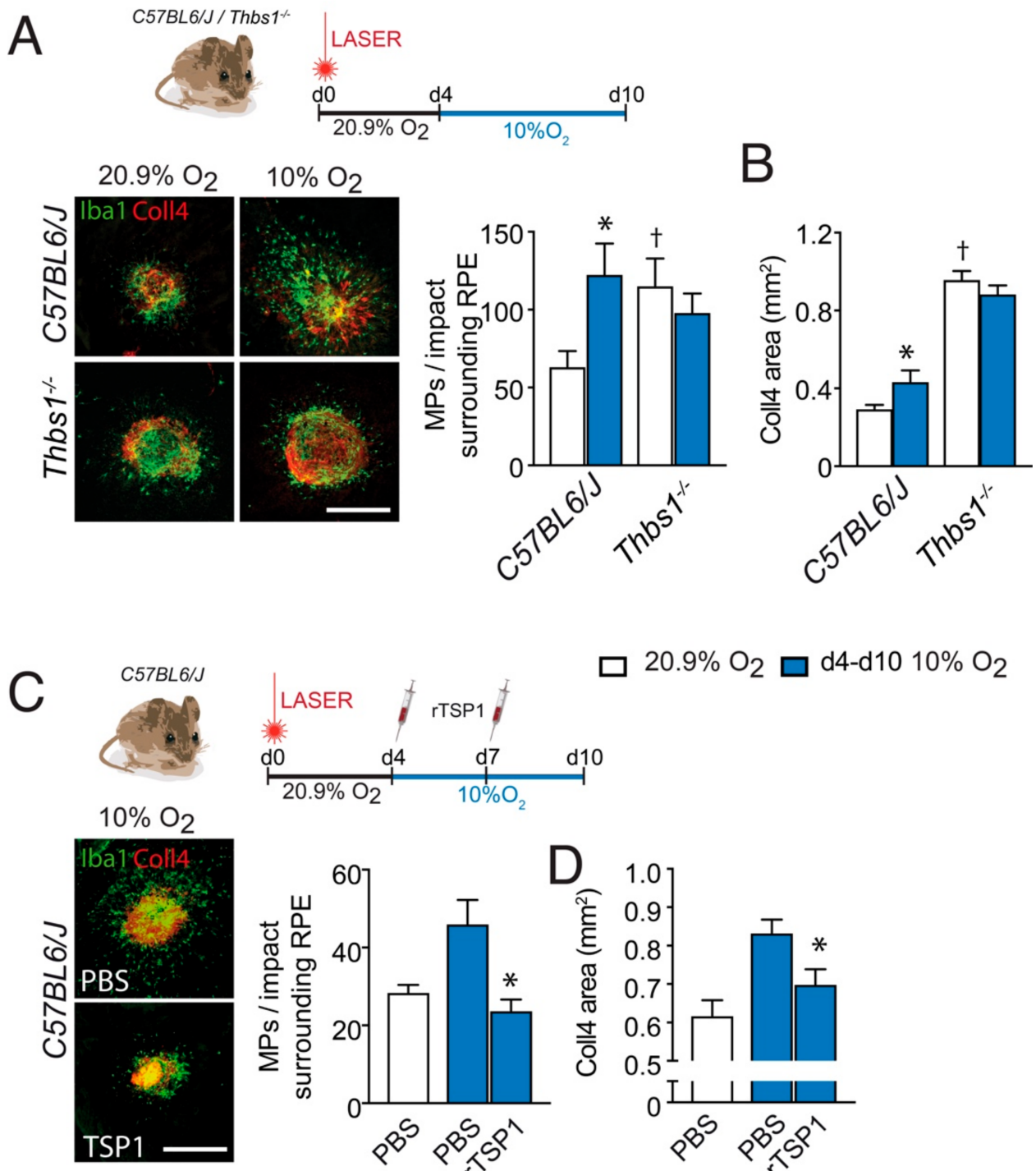

\section{$20.9 \% \mathrm{O}_{2} \square \mathrm{d} 4-\mathrm{d} 1010 \% \mathrm{O}_{2}$}

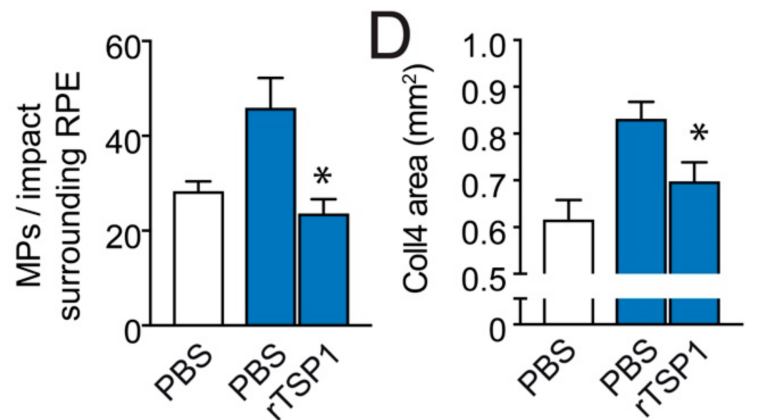

Figure 3. TSP-1 is necessary for hypoxia-induced inhibition of inflammation resolution after laserinjury. (A) Representative images of Collagen 4 (Coll4; red) and IBA-1 (green) immuno-stained $\mathrm{RPE} /$ choroid flatmounts of 10 day post laser-injured 2-month-old mice of the indicated strains, raised in $20.9 \%$ ambient $\mathrm{O}_{2}$ (normoxia, white columns) or exposed to $10 \% \mathrm{O}_{2}$ hypoxia ( $\mathrm{d} 4-\mathrm{d} 10$, blue columns) and quantification of subretinal IBA- $1^{+}$MPs per impact on the RPE counted at a distance of $0-500 \mu \mathrm{m}$ to Coll4 ${ }^{+} \mathrm{CNV}$ of the indicated strains $\left(n=9-10\right.$ eyes; ${ }^{*} p=0.0002{ }^{+} p=0.0114$; MannWhitney versus the normoxic C57BL6/J mice, hypoxic Thbs $1^{-/}$mice were not significantly different from hypoxic C57BL6/J mice). (B) Quantification of the Coll4 ${ }^{+} \mathrm{CNV}$ surface at $\mathrm{d} 10$ of normoxic and hypoxia-exposed (d4-d10) laser-injured mice of the indicated strains $\left(n=9-10\right.$ eyes; ${ }^{*} p<0.0001$; ${ }^{+} p=0.0114$; Mann-Whitney versus the normoxic C57BL6/J mice). (C) Representative images of Coll4 (red) and IBA-1 (green) immuno- stained RPE/choroid flatmounts of 10 day post laser-injured, 2 month-old, wildtype mice exposed to $10 \% \mathrm{O}_{2}$ hypoxia (d4-d10) that were intravitreally injected at $\mathrm{d} 4$ and $\mathrm{d} 7$ with $2 \mu \mathrm{L}$ of PBS, or $2 \mu \mathrm{L}$ of PBS containing recombinant Thrombospondin 1 protein (TSP-1, $10 \mu \mathrm{g} / \mathrm{mL}$ ) and the quantification of subretinal IBA- $1^{+}$MPs per impact on the RPE counted at a distance of $0-500 \mu \mathrm{m}$ to Coll4 ${ }^{+} \mathrm{CNV}$ at $\mathrm{d} 10\left(n=9-10\right.$ eyes; ${ }^{*} p=0.0001$; versus the hypoxic PBS-treated mice). (D) CNV surface at d10 of the treated, hypoxia-exposed (d4-d10), laser-injured mice ( $n=9-10$ eyes; ${ }^{*} p=0.00143$; Mann-Whitney versus the hypoxic PBS-treated mice). All values are reported as mean \pm SEM. PBS: Phosphate buffered saline; rTSP-1: Recombinant thrombospondin 1 ; scale bar $=400 \mu \mathrm{m}$. 


\section{Discussion}

There are many reasons to suspect that local hypoxia plays a role in the pathogenesis of AMD: drusen and pseudo-drusen deposits increase the distance of the choriocapillaries to the photoreceptors, the choriocapillary blood flow is reduced in intermediate and late $\mathrm{AMD}$, and $\mathrm{CNV}$ is preceded by choriocapillary dropout [9]. Intriguingly, there might also be a role for systemic hypoxia as AMD is associated with hypertension, atherosclerosis and cardiovascular disease [10] that are all associated with systemic hypoxia [12]. Hypoxic preactivation of circulating Mos [13] could thereby promote subretinal chronic inflammation and AMD. We here show that hypoxic ambient air $\left(10 \% \mathrm{O}_{2}\right)$ was sufficient to increase the infiltration induced by laser injury in vivo. Experimental mice were exposed to not more than six days of hypoxia in either the "recruitment"- or "resolution"-phase, as our preliminary data showed that longer-term exposition to $10 \%$ ambient $\mathrm{O}_{2}$, air had only marginal effects on subretinal inflammation and CNV likely because these young, healthy mice adapt to experimental hypoxia by erythrocytosis [30] similar to altitude adaptation. Interestingly, hypoxia increased the subretinal MP population that accumulates in the surrounding of the injury, in direct contact with the immune-suppressive RPE, but not in the number of MPs directly adjacent to the endothelial cells of the CNV. Hypoxic exposure following the peak infiltration at $\mathrm{d} 4$ when inflammation resolution occurs in room-air, completely prevented the decrease of MP numbers and let to a further increase of the lesionsurrounding MPs at d10 that was accompanied by more sizeable CNV formation (Figure 1). These results suggested that RPE-induced MP elimination might be affected during hypoxia. We previously showed that MPs are quickly eliminated by the RPE in adoptive transfer experiments of Mo and MCs to the subretinal space and in Mo-RPE cocultures in vitro, and that their activation, due to Cx3cr1-deficiency, exogenous APOE, HTRA1, or LPS, significantly increased their resistance to RPE-induced death $[4,5,15,16]$. Similarly, we here show that hypoxia significantly reduced RPE-induced human Mo elimination in co-culture in vitro and that this effect was due to an effect of hypoxia on Mos rather than the RPE (Figure 1).

Hypoxia can activate macrophages via the transcription factors hypoxia-inducible factor $1 \alpha$ and $2 \alpha$ (HIF- $1 \alpha$, HIF- $2 \alpha$ ), NF-kB and AP-1 that is formed by c-Jun and c-Fos [31-33]. Intriguingly, the regimen also led to a significant decrease in Thbs1- transcripts in Mo (Figure 2). These alterations might be due to c-jun, which is increased by hypoxia [34] and represses Thbs1-tanscription [35].

We recently demonstrated that TSP-1 activation of CD47 sensitizes infiltrating subretinal MPs to RPE induced death and elimination $[4,5]$. To test whether the hypoxia induced deregulation of TSP-1 could be responsible for the inhibition of the elimination of MPs in contact with the RPE we first compared post-injury inflammation resolution in normoxic and hypoxic Thbs1 ${ }^{-/-}$-mice to wildtype animals. At $\mathrm{d} 10 \mathrm{room}$ air raised Thbs $1^{-/-}$-mice, revealed a significantly greater number of subretinal MPs in accordance with its role in subretinal MP elimination others and we previously described [4-6] (Figure 3). The increased inflammation in Thbs $1^{-/-}$-mice was accompanied by increased CNV corroborating previous results [5-7]. Contrary to wildtype mice, hypoxic conditions failed to alter subretinal MP infiltration or CNV in Thbs1-/-- mice, showing that it is strictly necessary for the pathogenic effect of systemic hypoxia. Indeed, when we locally replaced TSP-1 in laser-injured mice, we were able to prevent the exaggerated neuro-inflammation and neovascularization induced by the systemic hypoxia (Figure 3).

In our experiments we exposed the mice to relatively short periods (4 to 6 days) of constant $10 \% \mathrm{O}_{2}$ hypoxia. This experimental design allowed us to apply hypoxia separately to the "recruitment"- or "resolution"-phase. Ten percent $\mathrm{O}_{2}$ constant hypoxia corresponds to the $\mathrm{O}_{2}$ partial pressure on Kilimanjaro (5895 m, 5 January 2021: https: //hypoxico.com/altitude-to-oxygen-chart/) that tourists frequently climb without oxygen supplementation, and can therefore be considered severe, but not extreme. Interestingly, a recent analysis of a cohort of 67786 sleep apnea syndrome (SAS) patients, has shown a strong association of chronic systemic intermittent hypoxemia that characterizes this 
condition, with wet AMD [36]. Although chronic intermittent hypoxia differs significantly from the chronic hypoxia in emphysema or our experimental conditions, it is interesting to note that SAS patients also display reduced serum TSP-1 levels [37] and we observed decreased TSP-1 transcription under hypoxic conditions in our experiments. Future studies using intermittent hypoxia and chronic hypoxia models are needed to determine similarities and differences between the two and also help to understand whether other AMD-risk factors such as age or genetic predispositions impede the adaptation to chronic hypoxia that we observed in young mice and which might prevent AMD in younger patients with chronic hypoxia.

Taken together, our study shows that systemic hypoxia leads to the activation of monocytes, the deregulation of TSP-1 expression and ensuing increased subretinal inflammation and production of pathogenic cytokines. Our study provides rationale for the implication of hypoxia and in particular systemic hypoxia in neuro-inflammation in AMD, and opens avenues toward therapies inhibiting pathogenic chronic inflammation in late AMD. Most importantly, our study shows that local TSP-1 injection can efficiently counter the effect of systemic hypoxia and inhibit pathogenic chronic inflammation in late AMD. A similar approach might also be beneficial in other inflammatory degenerative diseases that are associated with chronic systemic hypoxia, such as atherosclerosis [38] or Alzheimer's disease [39].

Author Contributions: Conceptualization, F.S., S.T.; Investigation, S.T., F.B. (Fanny Beguier), T.Y., S.A., C.R., F.B. (Frederic Blond), and J.B.C., Writing-Original Draft, F.S., S.T., B.B., C.D., X.G. and F.S.; Writing-Review and Editing, F.S., Funding Acquisition, F.S. and J.A.S.; Supervision, C.D., X.G. and F.S. All authors have read and agreed to the published version of the manuscript.

Funding: This work was supported by grants from Institut Nationale de la Santé et Recherche Médicale (INSERM), Agence Nationale de Recherche (ANR): ANR Osaging 2018 (ANR-18-CE14-0031-02); MACLEAR (ANR-15-CE14-0015-01), LABEX LIFESENSES [ANR-10-LABX-65], Programme Investissements d'Avenir Institut Hospitalo-Universitaire (IHU) FOReSIGHT (ANR-18-IAHU-01). Acknowledgement is made to the donors of the Macular Degeneration Research, a program of the BrightFocus Foundation, for support of this research (M2018096).

Institutional Review Board Statement: All experimental procedures involving animals were approved by the Ministere de l'éducation nationale, de l'enseignement supérieur et de la recherche (APAFIS\#2636-2015110914346299v2.

Acknowledgments: We wish to thank Luisa Riancho the platform of cellular and tissular phenotyping of the IdV, and A. Potey and M. Lechuga from the high-throughput screening platform of the IdV, supported by Department de Paris and by Region Ile de France.

Conflicts of Interest: The authors declare that they have no conflict of interest.

\section{References}

1. Sarks, S.H. Ageing and degeneration in the macular region: A clinico-pathological study. Br. J. Ophthalmol. 1976, 60, 324-341. [CrossRef]

2. Guillonneau, X.; Eandi, C.M.; Paques, M.; Sahel, J.A.; Sapieha, P.; Sennlaub, F. On phagocytes and macular degeneration. Prog. Retin Eye Res. 2017, 61, 98-128. [CrossRef]

3. Sennlaub, F.; Auvynet, C.; Calippe, B.; Lavalette, S.; Poupel, L.; Hu, S.J.; Dominguez, E.; Camelo, S.; Levy, O.; Guyon, E.; et al. CCR2(+) monocytes infiltrate atrophic lesions in age-related macular disease and mediate photoreceptor degeneration in experimental subretinal inflammation in Cx3cr1 deficient mice. EMBO Mo.l Med. 2013, 5, 1775-1793. [CrossRef]

4. Calippe, B.; Augustin, S.; Beguier, F.; Charles-Messance, H.; Poupel, L.; Conart, J.B.; Hu, S.J.; Lavalette, S.; Fauvet, A.; Rayes, J.; et al. Complement Factor H Inhibits CD47-Mediated Resolution of Inflammation. Immunity 2017, 46, 261-272. [CrossRef] [PubMed]

5. Beguier, F.; Housset, M.; Roubeix, C.; Augustin, S.; Zagar, Y.; Nous, C.; Mathis, T.; Eandi, C.; Benchaboune, M.; Drame-Maigne, A.; et al. The 10q26 Risk Haplotype of Age-Related Macular Degeneration Aggravates Subretinal Inflammation by Impairing Monocyte Elimination. Immunity 2020, 53, 429-441.e8. [CrossRef] [PubMed]

6. Ng, T.F.; Turpie, B.; Masli, S. Thrombospondin-1-mediated regulation of microglia activation after retinal injury. Investig. Ophthalmol. Vis. Sci. 2009, 50, 5472-5478. [CrossRef] 
7. Wang, S.; Sorenson, C.M.; Sheibani, N. Lack of thrombospondin 1 and exacerbation of choroidal neovascularization. Arch. Ophthalmol. 2012, 130, 615-620. [CrossRef] [PubMed]

8. Blasiak, J.; Petrovski, G.; Vereb, Z.; Facsko, A.; Kaarniranta, K. Oxidative stress, hypoxia, and autophagy in the neovascular processes of age-related macular degeneration. Biomed Res. Int. 2014, 2014, 768026. [CrossRef]

9. Stefansson, E.; Geirsdottir, A.; Sigurdsson, H. Metabolic physiology in age related macular degeneration. Prog Retin Eye Res 2011, 30, 72-80. [CrossRef]

10. Chakravarthy, U.; Wong, T.Y.; Fletcher, A.; Piault, E.; Evans, C.; Zlateva, G.; Buggage, R.; Pleil, A.; Mitchell, P. Clinical risk factors for age-related macular degeneration: A systematic review and meta-analysis. BMC Ophthalmol. 2010, 10, 31. [CrossRef]

11. Klein, R.; Klein, B.E.; Tomany, S.C.; Cruickshanks, K.J. The association of cardiovascular disease with the long-term incidence of age-related maculopathy: The Beaver Dam Eye Study. Ophthalmology 2003, 110, 1273-1280. [CrossRef]

12. Feigl, B. Age-related maculopathy-linking aetiology and pathophysiological changes to the ischaemia hypothesis. Prog. Retin Eye Res. 2009, 28, 63-86. [CrossRef] [PubMed]

13. Semenza, G.L. Hypoxia-inducible factors in physiology and medicine. Cell 2012, 148, 399-408. [CrossRef]

14. Lavalette, S.; Raoul, W.; Houssier, M.; Camelo, S.; Levy, O.; Calippe, B.; Jonet, L.; Behar-Cohen, F.; Chemtob, S.; Guillonneau, X.; et al. Interleukin-1beta inhibition prevents choroidal neovascularization and does not exacerbate photoreceptor degeneration. Am. J. Pathol. 2011, 178, 2416-2423. [CrossRef]

15. Levy, O.; Calippe, B.; Lavalette, S.; Hu, S.J.; Raoul, W.; Dominguez, E.; Housset, M.; Paques, M.; Sahel, J.A.; Bemelmans, A.P.; et al. Apolipoprotein E promotes subretinal mononuclear phagocyte survival and chronic inflammation in age-related macular degeneration. EMBO Mol. Med. 2015, 7, 211-226. [CrossRef] [PubMed]

16. Mathis, T.; Housset, M.; Eandi, C.; Beguier, F.; Touhami, S.; Reichman, S.; Augustin, S.; Gondouin, P.; Sahel, J.A.; Kodjikian, L.; et al. Activated monocytes resist elimination by retinal pigment epithelium and downregulate their OTX2 expression via TNF-alpha. Aging Cell 2017, 16, 173-182. [CrossRef] [PubMed]

17. Touhami, S.; Beguier, F.; Augustin, S.; Charles-Messance, H.; Vignaud, L.; Nandrot, E.F.; Reichman, S.; Forster, V.; Mathis, T.; Sahel, J.A.; et al. Chronic exposure to tumor necrosis factor alpha induces retinal pigment epithelium cell dedifferentiation. $J$. Neuroinflammation 2018, 15, 85. [CrossRef]

18. Charles-Messance, H.; Blot, G.; Couturier, A.; Vignaud, L.; Touhami, S.; Beguier, F.; Siqueiros, L.; Forster, V.; Barmo, N.; Augustin, S.; et al. IL-1beta induces rod degeneration through the disruption of retinal glutamate homeostasis. J. Neuroinflammation 2020, 17, 1. [CrossRef] [PubMed]

19. Levy, O.; Lavalette, S.; Hu, S.J.; Housset, M.; Raoul, W.; Eandi, C.; Sahel, J.A.; Sullivan, P.M.; Guillonneau, X.; Sennlaub, F. APOEisoforms control pathogenic subretinal inflammation in age related macular degeneration. J. Neurosci. 2015, 35, 13568-13576. [CrossRef]

20. Kim, D.; Langmead, B.; Salzberg, S.L. HISAT: A fast spliced aligner with low memory requirements. Nat. Methods 2015, 12, 357-360. [CrossRef]

21. Love, M.I.; Huber, W.; Anders, S. Moderated estimation of fold change and dispersion for RNA-seq data with DESeq2. Genome biology 2014, 15, 550. [CrossRef] [PubMed]

22. Dyer, N.P.; Shahrezaei, V.; Hebenstreit, D. LiBiNorm: An htseq-count analogue with improved normalisation of Smart-seq2 data and library preparation diagnostics. PeerJ 2019, 7, e6222. [CrossRef] [PubMed]

23. Caicedo, A.; Espinosa-Heidmann, D.G.; Pina, Y.; Hernandez, E.P.; Cousins, S.W. Blood-derived macrophages infiltrate the retina and activate Muller glial cells under experimental choroidal neovascularization. Exp. Eye Res. 2005, 81, 38-47. [CrossRef] [PubMed]

24. Sakurai, E.; Anand, A.; Ambati, B.K.; van Rooijen, N.; Ambati, J. Macrophage depletion inhibits experimental choroidal neovascularization. Investig. Ophthalmol. Vis. Sci. 2003, 44, 3578-3585. [CrossRef]

25. Tsutsumi, C.; Sonoda, K.H.; Egashira, K.; Qiao, H.; Hisatomi, T.; Nakao, S.; Ishibashi, M.; Charo, I.F.; Sakamoto, T.; Murata, T.; et al. The critical role of ocular-infiltrating macrophages in the development of choroidal neovascularization. J. Leukoc. Biol. 2003, 74, 25-32. [CrossRef]

26. Luhmann, U.F.; Robbie, S.; Munro, P.M.; Barker, S.E.; Duran, Y.; Luong, V.; Fitzke, F.W.; Bainbridge, J.; Ali, R.R.; Maclaren, R. The drusen-like phenotype in aging $\mathrm{Ccl} 2$ knockout mice is caused by an accelerated accumulation of swollen autofluorescent subretinal macrophages. Investig. Ophthalmol. Vis. Sci. 2009, 50, 5934-5943. [CrossRef] [PubMed]

27. Liu, J.; Copland, D.A.; Horie, S.; Wu, W.K.; Chen, M.; Xu, Y.; Paul Morgan, B.; Mack, M.; Xu, H.; Nicholson, L.B.; et al. Myeloid cells expressing VEGF and arginase-1 following uptake of damaged retinal pigment epithelium suggests potential mechanism that drives the onset of choroidal angiogenesis in mice. PLoS ONE 2013, 8, e72935. [CrossRef]

28. Robbie, S.J.; Georgiadis, A.; Barker, S.E.; Duran, Y.; Smith, A.J.; Ali, R.R.; Luhmann, U.F.; Bainbridge, J.W. Enhanced Ccl2-Ccr2 signaling drives more severe choroidal neovascularization with aging. Neurobiol. Aging 2016, 40, 110-119. [CrossRef]

29. Sakurai, E.; Taguchi, H.; Anand, A.; Ambati, B.K.; Gragoudas, E.S.; Miller, J.W.; Adamis, A.P.; Ambati, J. Targeted disruption of the CD18 or ICAM-1 gene inhibits choroidal neovascularization. Investig. Ophthalmol. Vis. Sci. 2003, 44, 2743-2749. [CrossRef] [PubMed]

30. Yu, A.Y.; Shimoda, L.A.; Iyer, N.V.; Huso, D.L.; Sun, X.; McWilliams, R.; Beaty, T.; Sham, J.S.; Wiener, C.M.; Sylvester, J.T.; et al. Impaired physiological responses to chronic hypoxia in mice partially deficient for hypoxia-inducible factor 1alpha. J. Clin. Investig. 1999, 103, 691-696. [CrossRef] [PubMed] 
31. Murdoch, C.; Muthana, M.; Lewis, C.E. Hypoxia regulates macrophage functions in inflammation. J. Immunol. 2005, 175, 6257-6263. [CrossRef]

32. Lewis, J.S.; Lee, J.A.; Underwood, J.C.; Harris, A.L.; Lewis, C.E. Macrophage responses to hypoxia: Relevance to disease mechanisms. J. Leukoc. Biol. 1999, 66, 889-900. [CrossRef]

33. Rahat, M.A.; Bitterman, H.; Lahat, N. Molecular mechanisms regulating macrophage response to hypoxia. Front/ Immunol/ 2011, 2, 45. [CrossRef]

34. Ausserer, W.A.; Bourrat-Floeck, B.; Green, C.J.; Laderoute, K.R.; Sutherland, R.M. Regulation of c-jun expression during hypoxic and low-glucose stress. Mol. Cell. Biol. 1994, 14, 5032-5042. [PubMed]

35. Mettouchi, A.; Cabon, F.; Montreau, N.; Vernier, P.; Mercier, G.; Blangy, D.; Tricoire, H.; Vigier, P.; Binetruy, B. SPARC and thrombospondin genes are repressed by the c-jun oncogene in rat embryo fibroblasts. EMBO J. 1994, 13, 5668-5678. [CrossRef]

36. Keenan, T.D.; Goldacre, R.; Goldacre, M.J. Associations between obstructive sleep apnoea, primary open angle glaucoma and age-related macular degeneration: Record linkage study. Br. J. Ophthalmol. 2017, 101, 155-159. [CrossRef] [PubMed]

37. Jurado-Gamez, B.; Gomez-Chaparro, J.L.; Munoz-Calero, M.; Serna Sanz, A.; Munoz-Cabrera, L.; Lopez-Barea, J.; Gozal, D. Serum proteomic changes in adults with obstructive sleep apnoea. J. Sleep Res. 2012, 21, 139-146. [CrossRef]

38. Pack, A.I.; Gislason, T. Obstructive sleep apnea and cardiovascular disease: A perspective and future directions. Prog. Cardiovasc. Dis. 2009, 51, 434-451. [CrossRef]

39. Daulatzai, M.A. Evidence of neurodegeneration in obstructive sleep apnea: Relationship between obstructive sleep apnea and cognitive dysfunction in the elderly. J. Neurosci. Res. 2015, 93, 1778-1794. [CrossRef] [PubMed] 